\title{
Visión retrospectiva de los 14 primeros años de investigación en materiales óxidos cerámicos superconductores en la UNMSM
}

\author{
J. C. González*1, L. De Los Santos Valladares ${ }^{2}$, A. Osorio ${ }^{3}$, J. Flores ${ }^{4}$ y A. Bustamante ${ }^{4}$
}

${ }^{1}$ Grupo de Investigación de Superficies, Intercaras y Láminas Delgadas, Instituto de Ciencia de Materiales de Sevilla, CSIC, Universidad de Sevilla, Calle Américo Vespucio 49, Isla de la Cartuja, 41092 Sevilla, España

${ }^{2}$ Cavendish Laboratory, Department of Physics, University of Cambridge, J. J. Thomson Ave., Cambridge CB3 OHE, Reino Unido

${ }^{3}$ Grupo Sol-Gel, Facultad de Química e Ing. Química, Universidad Nacional Mayor de San Marcos, Av. Venezuela s/n, Lima 1, Perú

${ }^{4}$ Laboratorio de Cerámicos y Nanomateriales, Facultad de Ciencias Físicas, Universidad Nacional Mayor de San Marcos, A.P. 14-0149, Lima 14, Perú

Recibido 30 noviembre 2011 - Aceptado 28 diciembre 2011

En abril de 2011 se celebró a nivel mundial los primeros 100 años del descubrimiento de la superconductividad. La superconductividad ha estado en el foco de la física del estado sólido fundamental, ciencias aplicadas e ingeniería. Hoy en día es posible utilizar los materiales superconductores en una primera generación de dispositivos superconductores y en un futuro cercano en trenes magnéticamente levitados, cables superconductores, computadoras cuánticas, nueva generación de teléfonos móviles, nuevos equipos de resonancia magnética, etc. El grupo de materiales de óxidos cerámicos superconductores perteneciente a la UNMSM hemos estudiado con sumo interés el compuesto superconductor $\mathrm{YBa}_{2} \mathrm{Cu}_{3} \mathrm{O}_{7}$, investigando la incorporación de distintos cationes y aniones en diversos sitios atómicos en la estructura, así también como en derivados de esta estructura, con el fin de comprender sus propiedades estructurales, químicas y superconductoras para proponer nuevos compuestos superconductores. Celebramos la primera centuria del descubrimiento de la superconductividad mediante una visión retrospectiva de nuestros resultados en los primeros 14 años de investigación.

Palabras claves: Superconductividad, materiales cerámicos superconductores, $\mathrm{YBa}_{2} \mathrm{Cu}_{3} \mathrm{O}_{7}$.

\section{A retrospective vision of the first 14 years on superconducting ceramic oxide} materials research in the UNMSM

In April 2011 took place in the world's first 100 years of the discovery of superconductivity. Superconductivity has been the focus of fundamental solid state physics, applied sciences and engineering. Today it is possible to use superconducting materials in a first generation of superconducting devices and in the near future in magnetically levitated trains, superconducting wires, quantum computers, new generation mobile phones, new MRI equipment, etc. The group of superconducting ceramic oxide materials belonging to the UNMSM have studied with great interest the $\mathrm{YBa}_{2} \mathrm{Cu}_{3} \mathrm{O}_{7}$ superconducting material, investigating the incorporation of different cations and anions in different atomic sites in the $\mathrm{YBa}_{2} \mathrm{Cu}_{3} \mathrm{O}_{7}$ structure, as well as derivatives of this structure, to understand their structural, chemical and superconducting properties, then proposing new superconducting compounds. We celebrate the first century of the discovery of superconductivity by a retrospective view of our results in the first 14 years of research.

Keywords: Superconductivity, superconducting ceramics, $\mathrm{YBa}_{2} \mathrm{Cu}_{3} \mathrm{O}_{7}$.

*juanc.gonzalez@icmse.csic.es 
El descubrimiento de la superconductividad en el mercurio se remonta a los inicios del siglo XX, exactamente el 08 de abril de 1911, por el físico holandés Heike Kammerling Onnes en Leiden, Holanda, justo después de conseguir licuar helio por primera vez, a bajas temperaturas. En 1913 gana el premio Nóbel por sus estudios del helio líquido. Dicho fenómeno se trata de una manifestación a escala macroscópica de un fenómeno cuántico y en el tiempo de Onnes no existía aún una teoría que explicase el fenómeno. Hubo que esperar los años más florecientes de la física del siglo XX, el desarrollo de la mecánica cuántica hasta el año 1957, para conseguir una teoría satisfactoria de un fenómeno tan sorprendente como la superconductividad.

Desde sus inicios la superconductividad se encuentra en la frontera de la innovación en el área de la ciencia y la tecnología [1]. En estos 100 años, desde el descubrimiento del fenómeno de la superconductividad, se han realizado progresos con gran dificultad, empezando por los superconductores convencionales, metales puros y aleaciones metálicas binarias o ternarias, hasta el descubrimiento de los superconductores cerámicos a base de óxidos de cobre, así como los compuestos orgánicos y los óxidos de hierro recientemente descubiertos, véase la línea de tiempo en la figura 1.

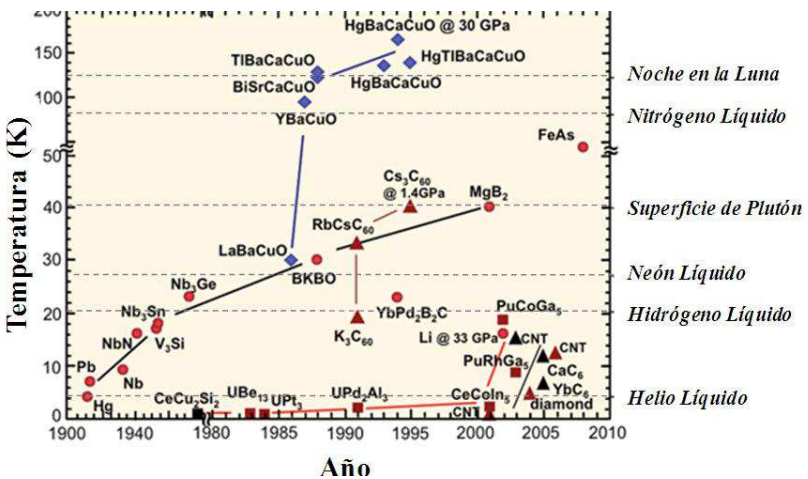

Figura 1: Línea de tiempo sobre el descubrimiento de los materiales superconductores.

Los especialistas comparan la llegada de la revolución tecnológica de los materiales superconductores con la forma en que los semiconductores revolucionaron el mundo a través del procesamiento computacional. Pero la superconductividad está aún llena de sorpresas, después de haber sido declarada un número de veces científicamente muerta, ésta ha regresado al foco de la investigación con nuevos efectos interesantes, desde el efecto Josephson en los superconductores de alta temperatura crítica hasta los superconductores basados en el hierro y los superconductores orgánicos.

Las aplicaciones de la superconductividad abarcan un área tan grande que ahora sería difícil de imaginar la ausencia de los dispositivos superconductores, en experimentos como los realizados en el Large Hadron Colli$\operatorname{der}$ [2] en el CERN y los experimentos de fusión nuclear como el ITER [3] podrían ser imposibles sin la superconductividad; cámaras astronómicas sin los detectores superconductores serían insensibles y las imágenes de resonancia magnética no serían una técnica estándar en los hospitales. Actualmente las cinco principales aplicaciones [4] de los materiales superconductores a nivel mundial son los alambres y las cintas superconductoras para el transporte de la energía, las imágenes de resonancia magnética, la generación de campos magnéticos de gran intensidad para su uso en la física de altas energías, las maquinas rotatorias y la detección de la materia oscura en astrofísica.

El fenómeno de la superconductividad aparece en ciertos materiales por debajo de una temperatura denominada temperatura crítica superconductora, $T_{c}$, caracterizada por una ausencia de resistencia eléctrica, $\rho=0$, y la exclusión de un débil campo magnético de su interior, $B_{\text {int }}=0$. Se distinguen dos tipos de materiales superconductores, los de baja y de alta temperatura crítica. El origen de la superconductividad en los de baja $T_{c}$ se puede explicar a partir de la formación de parejas de los portadores de cargas vía la interacción de excitaciones de la red, fonones. Cada electrón se acopla a un espectro de los fonones generando una región de atracción Coulombiana producida por los iones cargados positivamente, que atraen a su vez a otro electrón. La atracción es muy débil, pero como se demostró [5], aún una débil atracción es suficiente para crear estos estados electrónicos ligados. Además, las propiedades de transporte en estos materiales pueden ser comprendidos mediante la teoría desarrollada por Bardeen, Cooper y Schrieffer llamada de teoría BCS, en el año 1957. La teoría BCS [5] no especifica que los fonones median en la interacción, otras excitaciones también pueden servir para la formación de parejas electrónicas.

Por otro lado, los superconductores de alta $T_{c}$ aún no pueden ser descritos por el acoplamiento vía electrón-fonón. Últimamente se ha mostrado una evidencia [6] a favor de una distinta posibilidad. El acoplamiento electrónico vía paramagnones, u ondas de espín de corto alcance superpuestas a los espines de cobre con orden antiferromagnético subyacente. En la figura 2 se expone el diagrama de fase esquemático de los óxidos de cobre superconductores que existen sobre un rango contínuo junto con la fase aislante antiferromagnética no superconductora. El dopaje se refiere a la concentración de portadores de carga, huecos o electrones, en los óxidos de cobre no dopados y débilmente dopados poseen un orden antiferromagnético de largo alcance a bajas temperaturas. En el estado superconductor, el orden de largo alcance se rompe, pero las correlaciones de corto alcance aún permanecen. Estudios de resonancia 
magnética nuclear [7] han revelado que en los óxidos de cobre superconductores óptimamente dopados poseen correlaciones de espín en la escala de tres celdas unitarias. En algunos cupratos infradopados las correlaciones se extienden a 6 celdas unitarias. Este orden de corto alcance puede aún sustentar las ondas de espín, pero son amortiguadas. Habitualmente las ondas de espín de largo alcance son llamadas magnones, mientras que las ondas de espín amortiguadas son conocidas como paramagnones. Se sabe, experimentalmente, que los electrones se acoplan a las ondas de espín y teóricamente si los paramagnones existen en un amplio rango de energías, estos pueden producir la necesaria interacción de atracción [8] para inducir la superconductividad.

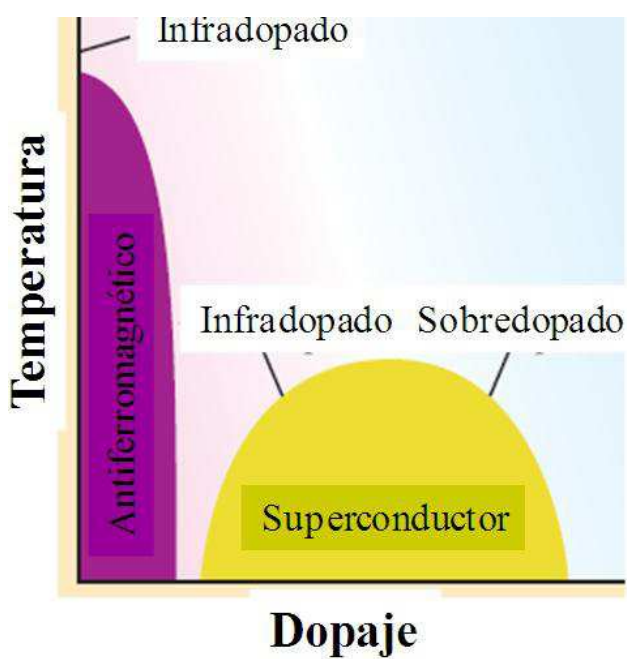

Figura 2: Diagrama de fase cualitativo de los óxidos de cobre superconductores

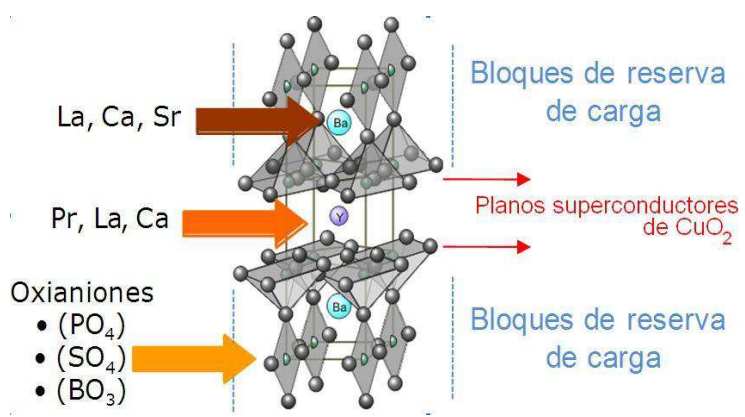

Figura 3: Bosquejo de la estructura del compuesto $\mathrm{YBa}_{2} \mathrm{Cu}_{3} \mathrm{O}_{7}$.

La química sustitutiva está muy relacionada con los materiales superconductores, ésta se realiza reemplazando cationes y/o aniones iso- o alio-valentes en la celda unitaria, la cual produce una presión química interna capaz de alterar la ocupancia, y la posición de los átomos en los bloques de reserva de carga o en los planos superconductores de $\mathrm{CuO}_{2}$. Esta presión química posee una directa influencia sobre la temperatura crítica superconductora, llevando al compuesto de un estado superconductor a un estado semiconductor o quizás, de mayor $T_{c}$. Un bosquejo de la estructura del $\mathrm{YBa}_{2} \mathrm{Cu}_{3} \mathrm{O}_{7}$ donde hemos realizado reemplazos iso- o alio-valentes de cationes y/o aniones se muestra en la figura 3. Una mejor descripción de los átomos que componen la estructura se encuentra descrita en la referencia [9].

El grupo de materiales cerámicos superconductores de la Facultad de Ciencias Físicas de la UNMSM ha venido estudiando intensamente el sistema $\mathrm{Y}-\mathrm{Ba}-\mathrm{Cu}-\mathrm{O}$ que presenta una $T_{c}=92 \mathrm{~K}$, la cual está por encima de la temperatura de ebullición del nitrógeno líquido, 77 $\mathrm{K}$, así como de las familias derivadas de esta estructura. En primer lugar, se ha estudiado la incorporación de átomos de $\mathrm{La}^{3+}, \mathrm{Ca}^{2+}, \mathrm{Pr}^{3+}$ en el sitio del $\mathrm{Y}^{3+}$, átomos de $\mathrm{La}^{3+}, \mathrm{Ca}^{2+}, \mathrm{Sr}^{2+}$ en el sitio del $\mathrm{Ba}^{2+}$ y los oxoaniones de $\left(\mathrm{PO}_{4}\right)^{3-},\left(\mathrm{BO}_{3}\right)^{2-},\left(\mathrm{SO}_{4}\right)^{3-}$ en el sitio del $\mathrm{Cu}(1)$ en las cadenas dentro de los bloques de reserva de carga y el efecto de los dopajes en las propiedades superconductoras. En segundo lugar, nos hemos interesado en el crecimiento de capas epitaxiales de $\mathrm{YBa}_{2} \mathrm{Cu}_{3} \mathrm{O}_{7}$ sobre sustratos monocristalinos con orientación [001] para fines tecnológicos como las cintas superconductoras y los dispositivos superconductores. Nos aunamos a las celebraciones mundiales de la primera centuria del descubrimiento de la superconductividad mediante una visión retrospectiva de nuestros resultados en los primeros 14 años de investigación en materiales óxidos cerámicos superconductores en la Universidad Nacional Mayor de San Marcos (UNMSM).

\section{Trabajos experimentales}

En nuestro grupo, las primeras síntesis de las cerámicas de alta $T_{c}$ fueron preparadas a través de la reacción de estado sólido. Posteriormente se realizaron mediante un proceso químico denominado sol-gel, estos métodos y sus posteriores tratamientos térmicos se encuentran claramente descritos en las referencias [10-16].

La caracterización estructural de los parámetros de red se llevó a cabo mediante la rutinaria difracción de rayos X en la geometría de Bragg-Brentano en difractómetros HZG, empresa de la ex-República Democrática Alemana y en un equipo Bruker D8 Focus y Panalitycal X'Pert Pro MPD Cu-K $\alpha$, con su posterior refinamiento Rietveld [17] de los parámetros estructurales. Además se realizó el cálculo de la valencia de los enlaces y de los diagramas de densidad electrónica de los electrones de valencia mediante programas como DBWS9807a y Fullprof. También hemos aplicado la difracción de rayos $\mathrm{X}$ con incidencia rasante, $\theta=1^{\circ}$ y la reflectometría de rayos $\mathrm{X}$ en capas delgadas de $\mathrm{YBa}_{2} \mathrm{Cu}_{3} \mathrm{O}_{7}$ crecidas sobre 
un monocristal de estructura perovskita de $\mathrm{SrTiO}_{3}$ y $\mathrm{LaAlO}_{3}$ con dirección [001] [18] para el estudio de las propiedades intercaras capa-sustrato. Las propiedades magnéticas fueron estudiadas en la región superconductora $T<T c$ y en la región normal $T>T_{c}$ mediante el uso de un magnetómetro con sensor Superconducting Quantum Inteference Device, SQUID, AC o DC de la firma Quantum Design en procesos de enfriamiento con campo aplicado field cooling, FC, o sin campo zero field cooling, ZFC.

Imágenes de microscopía electrónica de barrido y de transmisión fueron obtenidas mediante microscopios Phillips XL30 y FEI Phillips Tecnai 20, respectivamente, ambos microscopios acoplados con detectores Energy dispersive X-ray, EDX. Hemos obtenido patrones de difracción de electrones en muchas áreas seleccionadas para corroborar el contenido de cationes en los compuestos. Los espectros Raman fueron colectados mediante dos espectrómetros Raman, el primero es un triple espectrómetro Jobin Yvon T64000 equipado con un detector charge-coupled device, CCD, utilizando un láser de $\mathrm{Ar}^{+}$, con $\lambda=514.5 \mathrm{~nm}$ como excitación, y un espectrómetro RH800 Horiba Jovin-Yvon con detector CCD empleando un láser YAG con $\lambda=532 \mathrm{~nm}$ como excitación. Ambos espectrómetros tienen acoplado un microscopio metalográfico Olympus BX-41. Otras técnicas complementarias en colaboración con otras instituciones que están actualmente en ejecución son la espectroscopia Mössbauer, medidas de transporte eléctrico, I-V, a diferentes temperaturas, espectroscopia de infrarrojos, espectroscopia de fotoelectrones emitidos por rayos $\mathrm{X}, \mathrm{XPS}$.

A continuación, se describe nuestros resultados de los primeros 14 años de investigación dividida en tres períodos.

\section{Período 1994 - 2000}

Dos profesores principales, el Dr. Ángel Bustamante Domínguez y el Mg. Mateo Márquez Jacomé se reintegran a la Facultad de Ciencias Físicas de la UNMSM luego de haber realizado estudios de postgrado en el Centro Brasileiro de Pesquisas Físicas, en Rio de Janeiro, Brasil, trabajando con óxidos cerámicos superconductores $[19,20]$. En el año 1997, el Prof. Ángel Bustamante crea el grupo de investigación en materiales superconductores con la ayuda de los estudiantes de la maestría en física. Se inicia las primeras síntesis de óxidos de cobre mediante la reacción de estado sólido, investigando el sistema $\mathrm{Y}-\mathrm{Ba}-\mathrm{Cu}-\mathrm{O}$ mediante el dopaje de $\mathrm{Ca}^{2+}$ en el sitio de $\mathrm{Y}^{3}$ y los oxoaniones de $\left(\mathrm{PO}_{4}\right)^{3-}$, $\left(\mathrm{BO}_{3}\right)^{2-}$ en el sitio del $\mathrm{Cu}(1)$, los cuales generan números de coordinación 4 y 5 para el átomo de cobre $\mathrm{Cu}(2)$. Luego de hacer los cálculos estequiométricos correspondientes y de cuidadosos tratamientos térmicos, se logra que los compuestos resulten superconductores de alta $T_{c}$. Se halla la temperatura crítica superconductora de estos compuestos mediante las curvas de susceptibilidad magnética molar. Por otro lado, en la región normal se estudia el comportamiento Curie-Weiss, así como la cuantificación del magnetón de Bohr efectivo de los átomos de cobre en la estructura.

En 1998, se inicia el proyecto de investigación financiado por el Consejo Superior de Investigaciones de la UNMSM, denominado Preparación y Caracterización de las Cerámicas Superconductoras I siendo el responsable del proyecto el Prof. Angel Bustamante. En 1999, J. C. González, estudiante de la maestría en Física, obtiene el Young Investigator Travel Award [21] de la Universidad de Houston, EEUU. El líder del grupo de investigación, el Prof. Ángel Bustamante es distinguido por el Consejo Superior de Investigaciones de la UNMSM con el Mérito Científico por haber sido considerado el investigador científico más destacado de la Facultad de Ciencias Físicas. Se realizan las primeras exposiciones de los trabajos en congresos tales como el XIII Simposio Peruano de Física en Lima, en el 2000; el 6th International Conference of Superconductivity and High Temperature Superconductors en Houston, EEUU, en el 2000 y el Superconductors for Applications, Materials Properties and Devices en Rio de Janeiro, Brasil en el 2000. Seguidamente, se publican los primeros resultados de nuestras investigaciones en la Revista de Investigación de Física de la UNMSM [22-25] y en el Physica C: Superconductivity and its applications [12].

\section{Período 2001 - 2005}

El grupo de materiales superconductores de la UNMSM amplía el número de sus integrantes con estudiantes que cursan los últimos años del pregrado en Física de la Escuela Académico Profesional de Física de la Facultad de Ciencias Físicas. Se adiciona el estudio del sistema La-Ba-Cu-O a la química sustitutiva, se inicia también el estudio de la curva de irreversibilidad en el sistema $\mathrm{Nd}-\mathrm{Ba}-\mathrm{Cu}-\mathrm{O}$. Se aplica la espectrometria $\mu$-Raman para la detección de las fases precursoras que quedaron sin reaccionar, así como fases secundarias y espurias presentes, y la aparición de desorden catiónico o desorden en las cadenas de cobre oxígeno. Se estudia, además, la morfología de los granos superconductores mediante microscopio electrónico de barrido realizando una estadística de los tamaños de grano. Por otro lado, se decide iniciar otra vía de preparación utilizando métodos químicos [13]. Los métodos químicos permiten obtener compuestos homogéneos, con una buena estequiometría de cationes y aniones, además no requieren costosos sistemas de vacío, por lo cual son de bajo costo, y tienen principalmente una alta probabilidad de ser utilizados a escala industrial. La técnica sol-gel a 
diferencia de la ampliamente utilizada técnica de reacción de estado sólido permite una reacción química de los precursores. Por ejemplo en la preparación del compuesto $\mathrm{YBa}_{2} \mathrm{Cu}_{3} \mathrm{O}_{7}$ mediante dicha técnica, se logra una razón de cationes $\mathrm{Y}: \mathrm{Ba}: \mathrm{Cu}$ en proporción 1:2:3 a escala atómica. La solución precursora se convierte en un gel, para su posterior transformación en un óxido cerámico superconductor mediante la aplicación de un tratamiento térmico en atmósfera oxidante, usando un horno tubular programable LENTON.

Durante este periodo, se tiene la cobertura de los proyectos de investigación financiados como Preparación y caracterización de las cerámicas superconductoras III al IX, Preparación y caracterización del cerámico superconductor $\left[\mathrm{Bi}_{1-x} \mathrm{~Pb}_{x}\right] \mathrm{Sr}_{2} \mathrm{Ca}_{n-1} \mathrm{Cu}_{n} \mathrm{O}_{4+2 n}$ por el método de reacción de estado sólido y Preparación de óxidos cerámicos superconductores mediante el método de sol-gel, siendo los responsables los Profesores Ángel Bustamante, Jesús Flores y Ana María Osorio, respectivamente. En el 2004, Luis De Los Santos Valladares logra ocupar el primer lugar en el Primer Concurso Nacional de Tesis de Licenciatura, ECI 2004 [26]. Además, el Prof. Ángel Bustamante es distinguido nuevamente por el Consejo Superior de Investigaciones de la UNMSM con el Mérito Científico por haber sido considerado el investigador científico más destacado de la FCF. En este período se defienden cuatro tesis de Licenciatura [27-30] y una de maestría [31]. Los resultados de nuestras investigaciones son publicados en la Revista de Investigación de Física de la UNMSM [32,33], la Revista Peruana de Química e Ingeniería Química de la UNMSM $[13,15]$ y en el Physica C: Superconductivity and its Applications [34-38]. Se realizan exposiciones de los trabajos en congresos tales como el rth International Conference on Materials and Mechanism of Superconductivity and High Temperature Superconductors en Rio de Janeiro, Brasil, en el 2003; el XII Congreso Peruano de Química en Lima, en el 2004; el Spectroscopies in Novel Superconductors en Sitges, España, en el 2004, y en el XV Simposio Peruano de Física, Lima, 2005.

\section{Período 2006 - 2011}

El dominio en la preparación de materiales se intensifica con la técnica de sol-gel. Se inicia el crecimiento de capas de $\mathrm{YBa}_{2} \mathrm{Cu}_{3} \mathrm{O}_{7}$ sobre sustratos monocristalinos investigando las condiciones más óptimas para un crecimiento epitaxial. Se intensifica el estudio de la simetría local, el ordenamiento magnético, la cuantificación de las fracciones Meissner y blindaje diamagnético, la línea de irreversibilidad, la densidad de corriente crítica $J_{c}$, la valencia de los átomos en la estructura mediante el método de enlace de valencia, la densidad de electrones de valencia y la difracción de electrones en un área seleccionada para corroborar el contenido de cationes en el compuesto. Así como también, el grado de orientación de los granos superconductores en capas finas con respecto a la superficie del sustrato. Adicionalmente nuestro grupo viene estudiando la posibilidad de la fabricación de nano-transistores superconductores controlados por un campo magnético externo. Para esto ya se ha logrado funcionalizar nano-granos superconductores para que en un futuro próximo adherirlos a electrodos metálicos [39].

Durante este periodo se obtuvo el financiamiento para los proyectos Preparación y caracterización de las cerámicas superconductoras del IX al XIV, Influencia del dopaje sistemático en las propiedades superconductoras de los superconductores de alta temperatura y Preparación de óxidos cerámicos superconductores mediante el método de sol-gel, siendo el responsable el Prof. Ángel Bustamante, de los cinco primeros, y la Prof. Ana María Osorio, de los dos últimos. En el 2009, el Prof. Ángel Bustamante y la Prof. Ana M. Osorio son distinguidos por el Consejo Superior de Investigaciones de la UNMSM con el Mérito Científico por haber sido considerados los investigadores científicos más destacado de la Facultad de Ciencias Físicas y de la Facultad de Química e Ing. Química, respectivamente. En este período se defienden tres tesis de Licenciatura [40-42] y una de maestría [43]. Los resultados obtenidos en este periodo de investigación son publicados en la Revista de Investigación de Física de la UNMSM [44], en la Revista Peruana de Química e Ing. Química de la UNMSM [45-47], en la revista de la Sociedad Química del Perú [9,14,48-50], en el Advances in Science and Technology [16], en el Journal of Physics and Chemistry of Solids [51,52], en el Open Journal of Superconductivity [53], en el Japanese Journal of Applied Physics [39] y en el Hyperfine Interactions [54]. Se realizan las exposiciones de los trabajos en los congresos tales como el International Conferences on Modern Materials and Technologies en Sicilia, Italia, en el 2006; el IV Congreso Peruano de Tecnología y Ciencia de Materiales en Lima, en el 2006; el 8th International Conference on Materials and Mechanism of Superconductivity and High Temperature Superconductors en Dresden, Alemania, en el 2006; el XIII Congreso Peruano de Quimica en Lima, en el 2007; el XXIX Congreso Latinoamericano de Química en Cartagena de Indias, Colombia, 2010; el $43^{\text {rd }}$ IUPAC World Chemistry Congress en San Juan, Puerto Rico, en el 2011; el European Conference on Applied Superconductivity en La Haya, Holanda en el 2011 y el XX Simposio Peruano de Física en Tacna, en el 2011.

La investigación de materiales superconductores es una disciplina que requiere de proyectos financiados para poder solventar no sólo la compra de los materiales precursores sino las medidas de caracterización en 
costosos equipos y para el acceso a la información de trabajos de otros investigadores. Por citar un par de ejemplos, una medida de la susceptibilidad magnética de una muestra de $100 \mathrm{mg}$ en el rango de temperatura de 5 a $100 \mathrm{~K}$ usando un magnetómetro con sensor SQUID cuesta aproximadamente 300 Nuevos Soles, 75 euros; el acceso a la literatura científica, significa un costo elevado, medio millón de dólares americanos por año en suscripciones a revistas de investigación. En nuestro caso, dificultades como estas han sido parcialmente solucionadas mediante nuestra continua colaboración con distintos centros de investigación a nivel mundial. La figura 4 muestra las diferentes instituciones con las cuales tenemos colaboración, donde también nuestros integrantes han realizado o se encuentran realizando, sus estudios de doctorado, maestría, así como pasantías. Finalmente, la figura 5 expresa nuestra productividad en número de publicaciones arbitradas, comunicaciones a congresos y tesis generadas por nuestro grupo de investigación. Celebramos entonces los 100 primeros años de la superconductividad con el modesto aporte de alrededor de setenta publicaciones generada por nuestro grupo de investigación en esta área clave para el futuro de la sociedad.

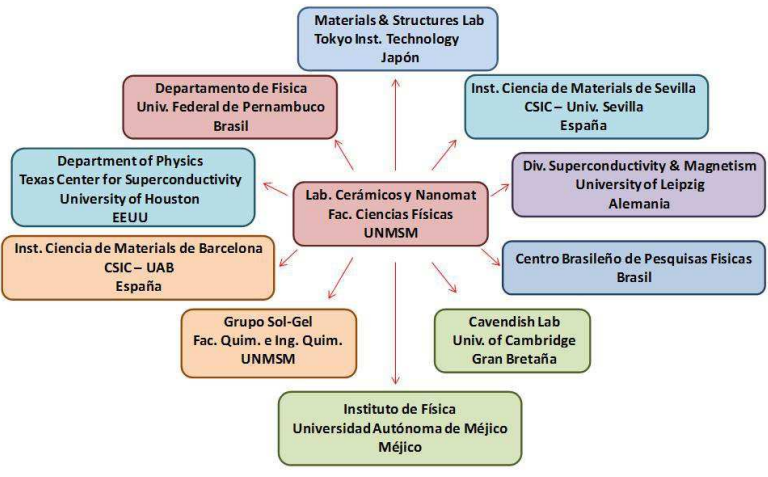

Figura 4: Relaciones interinstitucionales con otros centros de investigación.

\section{Perspectiva}

Después de 100 años la superconductividad todavía está vigorosa y se espera que se convierta en un éxito comercial para nuestra sociedad que cada vez requiere más y más energía. Su éxito depende de la fiabilidad y el precio de los sistemas superconductores incluyendo el enfriamiento, pero tiene la capacidad inherente de la eficiencia energética y compatibilidad medioambiental pudiéndose convertir en una tecnología clave para la sociedad del futuro. En la superconductividad casi nada parece imposible, en una serie de reuniones de expertos, están de acuerdo en que no existe un éxito intrínseco incluso para la superconductividad a temperatura ambiente.

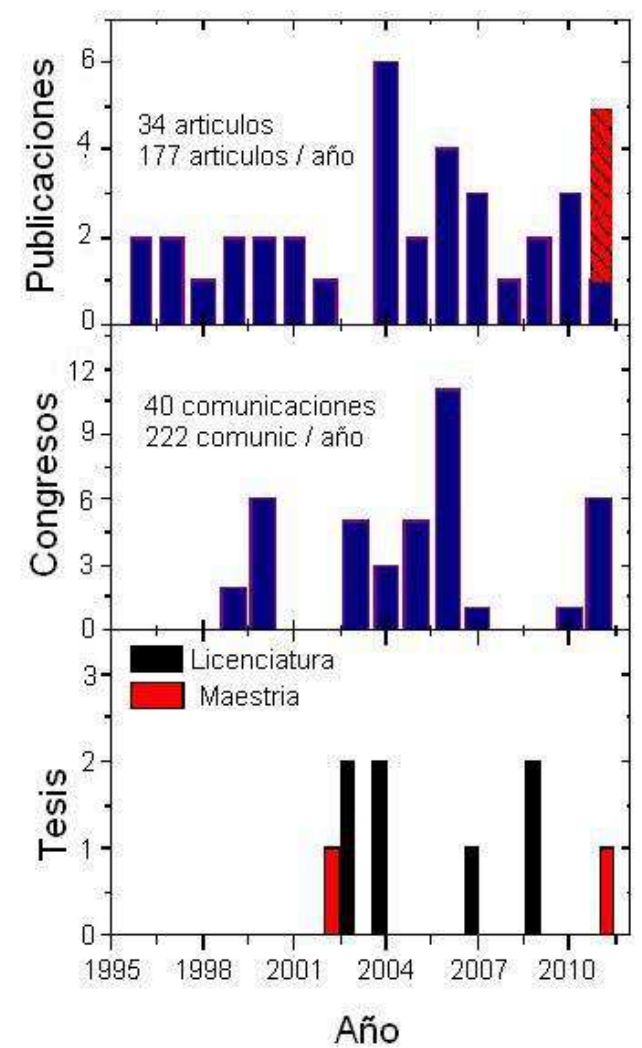

Figura 5: Producción científica en el éarea de superconductividad del grupo de materiales en óxidos cerámicos superconductores de la UNMSM.

\section{Conclusiones}

El hecho que los investigadores a nivel mundial continúen siendo sorprendidos con el descubrimiento de nuevos compuestos superconductores evidencia que aún no se tiene un firme control sobre lo que es necesario para conseguir este intrigante estado superconductor lo que posibilita seguir investigando en esta área. Sorprendentemente, aún después de 100 años del descubrimiento de la superconductividad vendrán tiempos muy excitantes donde las promesas de la superconductividad permanecen en un área activa en lo fundamental, así también como de investigación aplicada para las décadas venideras. Pero si bien es cierto que el grupo de investigación en materiales superconductores de la UNMSM pone el talento con un buen número de publicaciones indexadas, se requiere de inversión para desarrollar investigación en el país; más aún, si se quiere participar en proyectos multidisciplinarios y mayor presencia en la región. 


\section{Agradecimientos}

A. B. agradece al Vicerrectorado de Investigación y a la FCF de la UNMSM por el apoyo económico brindado desde el año 1998 hasta la fecha; A. M. O. y J. F. agradecen también al Vicerrectorado de Investigación y a la FQIQ y la FCF de la UNMSM, respectivamente, por el financiamiento económico brindado. J. C. G. agradece al CSIC de España por su contrato JAE-Doc
2009-2012 en el ICMSE-CSIC-US. L. D. L. S. V. agradece al programa ALBAN de la Unión Europea, Beca No. E06D101257PE y al Cambridge Overseas Trust por financiar sus estudios de Doctorado en la Universidad de Cambridge. Finalmente los autores agradecen a todos y cada uno de los investigadores en sus respectivas instituciones por la desinteresada colaboración con el grupo de materiales óxidos cerámicos superconductores perteneciente a la UNMSM.

\section{Referencias}

[1] J. McEntee; Chemistry World. 5, 42 (2008).

[2] http://Ihc.web.cern.ch/lhc/

[3] http://www.iter.org/proj

[4] P. Grantz; Phys. World 24, 3 (2011).

[5] J. Bardeen, L. N. Cooper y J. R. Schrieffer; Phys. Rev. 106, 162 (1957).

[6] M. Le Tacon, G. Ghiringhelli, J. Chaloupka, M. Moretti Sala, V. Hinkov, M. W. Haverkort, M. Minola, M. Bakr, K. J. Zhou, S. Blanco-Canosa, C. Monney, Y. T. Song, G. L. Sun, C. T. Lin, G. M. De Luca, M. Salluzzo, G. Khaliullin, T. Schmitt, L. Braicovich y B. Keimer; Nature Phys. 7, 725 (2011).

[7] H. Alloul, J. Bobroff, M. Gabay y P. J. Hirschfeld; Rev. Mod. Phys. 81, 45 (2009).

[8] D. J. Scalapino; Phys. Rep. 250, 329 (1995).

[9] J. C. González y A. Osorio; Rev. Soc. Quím. Perú. 74, 298 (2008).

[10] A. Bustamante, R. B. Scorzelli, E. BaggioSaitovitch, B. Giordanengo y M. Elmassalami; Physica C 235-240, 803 (1994).

[11] A. Bustamante, R. B. Scorzelli y E. Baggio Saitovitch; Hyperf. Inter. 110, 17 (1997).

[12] J. C. González, A. Bustamante, D. Landinez y J. Albino Aguiar; Physica C 341-348, 637 (2000).

[13] A. Bustamante, A. Osorio, J. C. González, M. H. Carhuancho, N. Salas, L. De los Santos, N. De la Cruz y A. Díaz; Rev. Per. Quim Ing. Quim. 7, 3 (2004).

[14] J. Feijóo, A. Osorio, A. Bustamante, L. De los Santos Valladares, A. Castro, M. H. Carhuancho y R. Aguirre; Rev. Soc. Quím. Perú 73, 208 (2007).
[15] A. Osorio, A. Bustamante, J. Feijoo, M. Bravo, J. C. González, R. Aguirre, M. Carhuancho, O. Cornejo, O. Ninan, E. Becerra, R. Lengua, J. Suarez y M. Zorrilla; Rev. Per. Quim. Ing. Quim. 8, 7 (2005).

[16] A. Bustamante, A. Osorio, L. De Los Santos, H. Carhuancho, J. C. González, G. Cernicchiario y J. Feijoo; Advances in Science and Technology 47, 37 (2006).

[17] R. A. Young; Introduction to the Rietveld Method, en The Rietveld method, Oxford University Press, Oxford (1993).

[18] A. Bustamante, A. Osorio. L. León, J. García, J. Flores, M. Pillaca, L. De Los Santos, D.W. Lee y J.C. González; European Conference on Applied Superconductivity (EUCAS 2011), abstract booklet, The Hague, The Netherlands, September (2011).

[19] Ángel Bustamante Domínguez; Tesis Doctoral, CBPF, Rio de Janeiro, Brasil (1995).

[20] Mateo A. Márquez Jácome; Tesis de Maestría, CBPF, Río de Janeiro, Brasil (1993).

[21] J. C. González; Young Investigator Travel Award (YITA-1999), Conference Secretariat, Texas Center for Superconductivity, University of Houston, Houston, Texas, USA (1999).

[22] A. Bustamante, J. Albino y D. Landinez; Rev. Inv. Fis. 1, 26 (1998).

[23] D. Sánchez, M. Márquez, S. García, E. BaggioSaitovitch; Rev. Inv. Fis. 2, 27 (1999).

[24] J. C. González, D. Landinez, J. Albino y A. Bustamante; Rev. Inv. Fis. 3, 24 (2000).

[25] Angel Bustamante Domínguez, R. B. Scorzelli y E. Baggio Saitovitch. Hyperf. Interact. 110, 17 (1997).

[26] L. De los Santos Valladares; Primer puesto en el Primer Concurso Nacional de Tesis de Licenciatura, organizado por el ECI-v2004 y el CONCYTEC, (2004). 
[27] L. De Los Santos; Tesis de Licenciatura, EAPFFCF-UNMSM, Lima (2003).

[28] Bram L. Willems; Tesis de Licenciatura, EAPFFCF-UNMSM, Lima, (2003).

[29] Richard Bellido; Tesis de Licenciatura, EAPFFCF-UNMSM, Lima (2004).

[30] Melchor LLosa; Tesis de Licenciatura, EAPF-FCFUNMSM, Lima (2004).

[31] J. C. González; Tesis de Maestría, UPG-FCFUNMSM, Lima (2002).

[32] A. Bustamante, L. De Los Santos, J. Flores, R. Lozano, J. Palomino y G. Gernicchiario; Rev. Inv. Fis. 7, 30 (2004).

[33] M. Llosa, L. De Los Santos, N. De La Cruz y A. Bustamante; Rev. Inv. Fis. 7, 34 (2004).

[34] J. C. González, A. Bustamante, D. Landinez y J. Albino; Physica C 354, 375 (2001).

[35] J. C. González, J. Albino, S. Quezada, L.M. Dezanetie, C. W. Chu y A. Bustamante; Physica C 354, 441 (2001).

[36] V. H. Barinotto, B. L. Willems, A. Bustamante, L. De Los Santos y J. C. González; Physica C 408410, 58 (2004).

[37] L. De Los Santos, A. Bustamante, J. Flores y J. C. González; Physica C 408-410, 44 (2004).

[38] A. Bustamante, R. Bellido, L. De Los Santos y J. C. González; Physica C 408-410, 884 (2004).

[39] L. De Los Santos Valladares, A. Bustamante, J. Llandro, S. Suzuki, T. Mitrelias, R. Bellido Quispe, C. H. W. Barnes y Y. Majima; Jpn. J. Appl. Phys. 48, 093102 (2010).

[40] L. Flores Ramos; Tesis de Licenciatura, EAPFFCF-UNMSM, Lima (2007).

[41] J. Feijoo; Tesis de Licenciatura, EAPF-FCFUNMSM, Lima (2009).
[42] G. Yactayo; Tesis de Licenciatura, EAPF-FCFUNMSM, Lima (2009).

[43] L. De Los Santos; Tesis de Maestría, UPG-FCFUNMSM, Lima (2011).

[44] J. C. González, A. Osorio, A. Bustamante; Rev. Inv. Fis. 12, 6 (2009).

[45] J. C. González, A. Osorio, R. Marin, A. Castro, J. Sandivar, A. Rodríguez y R. Flores; Rev. Per. Quim. Ing. Quim. 8, 12 (2006).

[46] J. C. González, A. Osorio y A. Bustamante; Rev. Per. Quim. Ing. Quim. 9, 11 (2006).

[47] L. De Los Santos Valladares, D. Acosta Najarro, A. Bustamante Domínguez, C. R. Magaña, R. Bellido Quispe, J. Flores Santibañez y A. Díaz Sandoval; Rev. Per. Quím. Ing. Quím. 10, 24 (2007).

[48] J. C. González, A. Osorio, A. Bustamante; Aceptado en Rev. Soc. Quím. Peru (2011).

[49] J. C. González, L. De los Santos, J. Feijoo, A. Osorio, A. Bustamante; Rev. Soc. Quim. Peru 75, 422 (2009).

[50] J. Feijoo, A. Bustamante L. De los Santos, B. L. Willems, A. Osorio, y J.C. González; Rev. Soc. Quím. Peru 76, 43 (2010).

[51] L. De Los Santos, A. Bustamante, J. C. González, J. Flores y X. Obradors; J. Phys. Chem. Solids. 67, 605 (2006).

[52] A. Bustamante, L. De Los Santos, B. L. Willems, V. H. Barinotto, J. C. González y X. Obradors; J. Phys. Chem. Solids. 67, 594 (2006).

[53] L. De Los Santos, A. Bustamante, J. C. González, J. Feijoo, A. Osorio, T. Mitrelias, Y. Majima y C. Barnes; The Open Superconductors Journal 2, 19 (2010).

[54] A. Bustamante, L. De Los Santos, J. Flores, C. H. W. Barnes y Y. Majima; Hyperfine Interactions 203, 119 (2011). 